\title{
Umbilical cord blood-derived microglia-like cells to model COVID-19 exposure
}

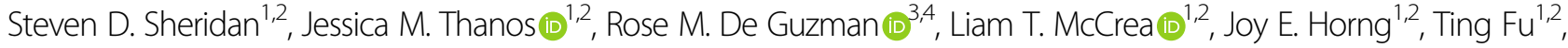
Carl M. Sellgren ${ }^{5}$, Roy H. Perlis (1, $\mathbb{1}^{1,2}$ and Andrea G. Edlow 3,4

\begin{abstract}
Microglia, the resident brain immune cells, play a critical role in normal brain development, and are impacted by the intrauterine environment, including maternal immune activation and inflammatory exposures. The COVID-19 pandemic presents a potential developmental immune challenge to the fetal brain, in the setting of maternal SARSCoV-2 infection with its attendant potential for cytokine production and, in severe cases, cytokine storming. There is currently no biomarker or model for in utero microglial priming and function that might aid in identifying the neonates and children most vulnerable to neurodevelopmental morbidity, as microglia remain inaccessible in fetal life and after birth. This study aimed to generate patient-derived microglial-like cell models unique to each neonate from reprogrammed umbilical cord blood mononuclear cells, adapting and extending a novel methodology previously validated for adult peripheral blood mononuclear cells. We demonstrate that umbilical cord blood mononuclear cells can be used to create microglial-like cell models morphologically and functionally similar to microglia observed in vivo. We illustrate the application of this approach by generating microglia from cells exposed and unexposed to maternal SARS-CoV-2 infection. Our ability to create personalized neonatal models of fetal brain immune programming enables non-invasive insights into fetal brain development and potential childhood neurodevelopmental vulnerabilities for a range of maternal exposures, including COVID-19.
\end{abstract}

\section{Introduction}

Maternal immune activation can result from exposures ranging from metabolic conditions to stress and infection, with potential in utero consequences to the developing fetus ${ }^{1-6}$. In particular, epidemiologic studies strongly suggest that maternal viral and bacterial infections in pregnancy may be associated with adverse neurodevelopmental outcomes in offspring, particularly autism spectrum disorders, schizophrenia, and cerebral palsy, but potentially including mood and anxiety disorders as well ${ }^{1-3,7-9}$. For instance, individuals who were fetuses during the 1957 influenza pandemic had a significantly increased risk for being hospitalized for

Correspondence: Roy H. Perlis (rperlis@mgh.harvard.edu) or

Andrea G. Edlow (aedlow@mgh.harvard.edu)

${ }^{1}$ Department of Psychiatry, Harvard Medical School, Boston, MA, USA

${ }^{2}$ Center for Genomic Medicine and Department of Psychiatry, Massachusetts

General Hospital, Boston, MA, USA

Full list of author information is available at the end of the article

These authors contributed equally: Steven D. Sheridan, Jessica M. Thanos schizophrenia as an adult ${ }^{10}$. The magnitude of these effects varies, but their consistency is difficult to ignore. Although mechanisms underlying neurodevelopmental morbidity in offspring remain unclear, microglial priming toward a pro-inflammatory phenotype with consequent altered synaptic pruning has been suggested as a candidate mechanism ${ }^{11-17}$.

Microglia, brain-resident tissue macrophages, play a key role in normal neurodevelopment by modulating synaptic pruning, neurogenesis, phagocytosis of apoptotic cells, and regulation of synaptic plasticity ${ }^{18-21}$. Fetal yolk sac-derived macrophages are the progenitors for the permanent pool of brain microglia throughout an individual's lifetime ${ }^{22-25}$. As such, inappropriate fetal microglial priming ("trained immunity"26) due to in utero immune activation may have lifelong neurodevelopmental consequences. The central role of mononuclear cells, including macrophages, in COVID-19 pathogenesis ${ }^{27}$ suggests that the potential risk to exposed fetal microglia requires investigation.

\section{(-) The Author(s) 2021}

(c) (i) Open Access This article is licensed under a Creative Commons Attribution 4.0 International License, which permits use, sharing, adaptation, distribution and reproduction cc) in any medium or format, as long as you give appropriate credit to the original author(s) and the source, provide a link to the Creative Commons license, and indicate if changes were made. The images or other third party material in this article are included in the article's Creative Commons license, unless indicated otherwise in a credit line to the material. If material is not included in the article's Creative Commons license and your intended use is not permitted by statutory regulation or exceeds the permitted use, you will need to obtain permission directly from the copyright holder. To view a copy of this license, visit http://creativecommons.org/licenses/by/4.0/. 
We have previously developed and validated adult patient-specific models of microglia-mediated pruning by reprogramming induced microglial cells from human peripheral blood mononuclear cells (PBMCs), and assaying them with isolated synapses (synaptosomes) derived from neural cultures differentiated from induced pluripotent stem cells (iPSCs) ${ }^{28,29}$. We demonstrated robust evidence of abnormalities in microglia and synaptosomes from individuals with schizophrenia, shown to be complement-dependent through a C3 receptor neutralizing antibody and rescued in a dose-responsive fashion with a small molecule probe ${ }^{28}$. Other groups have similarly applied in vitro synaptic pruning assays to provide insight into the pathogenesis of autism spectrum disorder and neurodegenerative disorders ${ }^{30-32}$.

In this study, we investigated whether our validated reprogramming methods for adult PBMCs could be successfully adapted and applied to umbilical cord bloodderived mononuclear cells (CB-MNCs) from both SARSCoV-2 infected and uninfected pregnancies to create personalized models of fetal brain microglia. Such models could have a wide range of application in investigating effects of in utero exposure on neurodevelopment. To illustrate this application, we demonstrate successful induction of microglia-like cells (CB-iMGs) from CB$\mathrm{MNCs}$ from both infected and uninfected pregnancies.

\section{Materials/subjects and methods}

\section{Ethical statement}

This study was approved by the Partners Institutional Review Board. Informed consent was obtained from all participants.

\section{Isolation and preparation of mononuclear cells from umbilical cord blood (CB-MNCs)}

Umbilical cord blood was collected at the time of delivery into EDTA tubes for plasma and CB-MNC isolation. After spinning at $1000 \mathrm{~g}$ for $10 \mathrm{~min}$ to separate plasma, samples were processed for CB-MNC isolation using a Ficoll density gradient ${ }^{33}$. Briefly, blood was transferred into a $50 \mathrm{~mL}$ conical tube and then diluted to 1:1 ratio with Hanks' balanced salt solution without calcium or magnesium (HBSS minus). This diluted blood was then gently layered on top of Ficoll at 2:1 ratio (two volumes of blood diluted with HBSS minus to one volume Ficoll). The conical tube was then centrifuged at $1000 \mathrm{~g}$ for $30 \mathrm{~min}$ at room temperature with brake inactivated to allow layering of cellular components. The cloudy ring below the plasma and above the Ficoll (i.e. the CB-MNC layer) was collected and placed in a new $15 \mathrm{~mL}$ conical tube, with HBSS minus added to bring the volume to $15 \mathrm{~mL}$. This tube was then centrifuged at $330 \mathrm{~g}$ for $10 \mathrm{~min}$ with high brake. The supernatant was removed and the CB-MNC pellet was washed with HBSS minus and resuspended in $10 \mathrm{~mL}$
HBSS minus for counting. Cells were counted on a hemocytometer in a 1:10 dilution of trypan blue. Cells were frozen in freezing medium consisting of RPMI 1640 Medium with $1 \%$ penicillin-streptomycin, L-glutamine, $1 \%$ sodium pyruvate, $1 \%$ non-essential amino acids, $20 \%$ fetal bovine serum (FBS), and 10\% DMSO at 5-10 million cells/ vial, placed in a chilled Mr. Frosty, then into $-80^{\circ} \mathrm{C}$. The following day, CB-MNC cryovials were transferred to liquid nitrogen for long-term storage. Isolated cryopreserved adult peripheral blood mononuclear cells were obtained from a single healthy control donor by Vitrologic (https://vitrologic.com) cat\# MNC-300.

\section{Derivation of induced microglia-like cells from CB-MNCs by direct cytokine reprogramming}

iMGs were derived using previously described methods ${ }^{28,29}$ while CB-iMGs were derived from CB-MNCs with modifications as noted. Briefly, cryopreserved PBMCs or CBMNCs were rapidly thawed at $37^{\circ} \mathrm{C}$, diluted into media consisting of RPMI 1640 with 10\% FBS and 1\% penicillin/ streptomycin. The cell suspension was centrifuged at $300 \mathrm{~g}$ for $5 \mathrm{~min}$ at room temperature, with the brake off. After aspirating the supernatant, the cell pellet was resuspended in media, counted, and plated on Geltrex-coated 24-well plates at $1 \times 10^{6}$ cells per $0.5 \mathrm{~mL}$ per well. After cells were incubated at $37^{\circ} \mathrm{C}$ for $24 \mathrm{~h}$, the media was completely replaced with RPMI 1640 with GlutaMAX, 1\% penicillin-streptomycin, $100 \mathrm{ng} / \mathrm{mL}$ of human recombinant IL-34 (Peprotech), and $10 \mathrm{ng} / \mathrm{mL}$ of GM-CSF (Peprotech). Media was replaced on day 13 after the initial cytokine reprogramming, and realtime live cell imaging or immunocytochemistry was performed on day 14 .

\section{iPSC generation and neural differentiation for synaptosome isolation}

iPSCs were reprogrammed from fibroblasts and used to derive neural progenitor cells, which were differentiated into neural cultures, as previously described ${ }^{28,29}$. In brief, adult human fibroblasts were reprogrammed to iPSCs using non-integrating synthetic RNA pluripotency factors, expanded and cryopreserved by Cellular Reprogramming, Inc. (https://www.cellular-reprogramming.com). To initiate neural progenitor induction, iPSCs were cultured feeder-free in E8 medium (Gibco) on Geltrex-coated sixwell plates and passaged using $50 \mathrm{mM}$ EDTA and trituration with ROCK inhibitor $(10 \mathrm{mM}$ Thiazovivin; Stemgent). iPSCs were further purified using magneticactivated cell sorting with Tra-1-60 microbeads (Miltenyi Biotec) on LS columns as described by vendor. Neural progenitor cells (NPCs) were derived from these iPSCs using neurobasal medium (Thermo Fisher Scientific) with $1 \times$ Neural Induction Supplement (Thermo Fisher Scientific), expanded using a neural expansion medium, and purified by double sorting using MACS against CD271 
and CD133. NPCs were immunostained for markers, including Nestin, SOX1, SOX2, and Pax-6. Validated NPCs were seeded for neural differentiation on Geltrexcoated T1000 five-layer cell culture flasks (Millipore Sigma \# PFHYS1008) and grown in neuronal differentiation medium (Neurobasal media (Gibco \# 21103049) supplemented with $1 \times$ each (N2 supplement (Stemcell Technologies SCT \# 7156), B27 supplement without Vitamin A (Gibco \# 12587010), non-essential amino acids (NEAA Gibco \# 11140050), penn/strep), $1 \mu \mathrm{M}$ ascorbic acid, $10 \mathrm{ng} / \mathrm{mL}$ BDNF and GDNF (Peprotech), and $1 \mu \mathrm{g} /$ $\mathrm{mL}$ mouse laminin (Sigma \# L2020) for 8 weeks.

\section{Synaptosome isolation by sucrose gradient}

Synaptosome isolation by sucrose gradient was adapted for iPSC-derived differentiated neural cultures from previously described protocols ${ }^{34-36}$. First, media was aspirated from flasks and cells were washed or scraped with $1 \times$ gradient buffer (ice-cold $0.32 \mathrm{M}$ sucrose, $600 \mathrm{mg} / \mathrm{L}$ Tris, $1 \mathrm{mM} \mathrm{NaH} \mathrm{CO}_{3}, 1 \mathrm{mM}$ EDTA, pH 7.4 with added HALT protease inhibitor-Thermo Fisher \# 78442), homogenized using a dounce homogenizer and centrifuged at $700 \mathrm{~g}$ for $10 \mathrm{~min}$ at $4{ }^{\circ} \mathrm{C}$. The pellet was resuspended in $10 \mathrm{~mL}$ of $1 \times$ gradient buffer (transferred to a $30 \mathrm{~mL}$ and centrifuged at $15,000 \mathrm{~g}$ for $15 \mathrm{~min}$ at $4{ }^{\circ} \mathrm{C}$. The second pellet was resuspended in $1 \times$ gradient buffer and slowly added on top of a sucrose gradient with $1 \times$ gradient buffer containing $1.2 \mathrm{M}$ (bottom) and $0.85 \mathrm{M}$ (middle) sucrose layers. The gradient and cell mixture was centrifuged at 26,500 r.p.m. $(\sim 80,000 g)$ for $2 \mathrm{~h}$ at $4{ }^{\circ} \mathrm{C}$, with the brake set to "slow" so as not to disrupt the final bands. The mixtures were handled carefully and bands were inspected to confirm successful fractionation. The synaptosome band (in between 0.85 and $1.2 \mathrm{M}$ sucrose) was removed with a $5-\mathrm{mL}$ syringe and $19 \mathrm{Gx} 1 \frac{112^{\prime \prime}}{}$ needle and centrifuged at $20,000 \mathrm{~g}$ for $20 \mathrm{~min}$ at $4{ }^{\circ} \mathrm{C}$. The final pellet was resuspended in an appropriate volume of $1 \times$ gradient buffer with $1 \mathrm{mg} / \mathrm{mL}$ bovine serum albumin (BSA) with protease and phosphatase inhibitors, aliquoted and slowly frozen at $-80^{\circ} \mathrm{C}$. Protein concentration was measured by BCA, and synaptosomes were further analyzed by transmission electron microscopy for heterogeneity of size and morphology, and enrichment of presynaptic (synapsin, SNAP-25) and post-synaptic (PSD-95) markers was determined by western blot analysis as previously described ${ }^{28,29}$.

\section{Real-time live cell imaging of synaptosome phagocytosis by CB-iMGs}

Real-time live cell imaging of CB-iMGs was performed as previously described ${ }^{28,29}$. Briefly, cells were imaged on the IncuCyte ZOOM live imaging system (Essen Biosciences) while incubated at $37^{\circ} \mathrm{C}$ with $5 \% \mathrm{CO}_{2}$. Synaptosomes were sonicated and labeled with pHrodo Red SE
(Thermo Fisher Scientific) and added to CB-iMGs at $15 \mu \mathrm{g}$ total protein per well in 24-well plates. Phase contrast and red fluorescence channel images were taken at a resolution of $0.61 \mu \mathrm{m}$ per pixel every $45 \mathrm{~min}$ for a total of $315 \mathrm{~min}$. Images were exported as 16-bit grayscale files and analyzed using CellProfiler ${ }^{37}$ to quantify cells and phagocytized particles. CellProfiler pipeline description are included in Supplementary Materials.

\section{Immunocytochemistry and confocal microscopy}

iMGs were fixed with $4 \%$ paraformaldehyde in phosphate-buffered saline (PBS) for $15 \mathrm{~min}$ at $4{ }^{\circ} \mathrm{C}$, washed twice with PBS, blocked for $1 \mathrm{~h}$ with $5 \% \mathrm{FBS}$ and 0.3\% Triton-X (Sigma Aldrich) in PBS, and then washed three times with $1 \%$ FBS in PBS. Cells were incubated with primary antibodies in 5\% FBS and $0.1 \%$ Triton-X overnight at $4{ }^{\circ} \mathrm{C}$ (Anti-IBA1, 1:500; Abcam \#ab5076; Anti-CX3CR1, 1:100, Abcam ab8021; Anti-PU.1, 1:11,000, Abcam \#ab183327, and Anti-P2RY12, 1:100, Alomone Labs). Cells were then washed twice with $1 \%$ FBS in PBS and incubated in secondary antibodies (1:500) and Hoechst 33342 (1:5000) in 5\% FBS and 0.1\% Triton-X in PBS for $45 \mathrm{~min}$ at $4{ }^{\circ} \mathrm{C}$, light-protected. Cells were washed twice and imaged using the IN Cell Analyzer 6000 (Cytiva). CellProfiler pipeline description used for quantification of marker immunopositive cell percentages are included in Supplementary Materials.

\section{Quantitation of microglial marker gene expression by $q R T-P C R$}

Total RNA was extracted using the RNeasy Plus Micro Kit (Qiagen \# 74034) and treated with dsDNAse (Thermo Scientific \# EN0771) for $10 \mathrm{~min}$ at $37^{\circ} \mathrm{C}$ followed by heat inactivation for $5 \mathrm{~min}$ at $55^{\circ} \mathrm{C}$ in the presence of $10 \mathrm{mM}$ DTT. Forty-five nanograms of purified RNA was used for cDNA synthesis using the Superscript III First-Strand Synthesis System (Invitrogen \# 18080051). Generated cDNA was diluted $1: 3$ and $3 \mu \mathrm{L}$ were loaded into the quantitative PCR reaction containing TaqMan Fast Advanced Master Mix (Applied Biosystems \# 4444556) and the TaqMan Gene Expression Assays (1x) for both the gene of interest and the housekeeping gene (Applied Biosystems; IBA1: Hs00610419_g1, PU.1: Hs02786711_m1, P2RY12: Hs00224470_m1, TMEM119: Hs01938722_u1, RPLP0: Hs00420895_gH). All reactions were run in technical triplicates in a $10 \mu \mathrm{L}$ reaction volume in a 384-well plate with a Roche LightCycler 480 machine as follows: $50^{\circ} \mathrm{C}$ for $2 \mathrm{~min}, 95^{\circ} \mathrm{C}$ for $20 \mathrm{~s}$, then 40 cycles of $95^{\circ} \mathrm{C}$ for $1 \mathrm{~s}$ and $60{ }^{\circ} \mathrm{C}$ for $20 \mathrm{~s}$. Ct values over 35 were set to the baseline $\mathrm{Ct}$ of 35 given inconsistent detection that occurs with low transcript levels. Relative quantification of gene expression was normalized to the endogenous housekeeping gene (RPLP0) then to the experimental negative control (PBMCs, CB-MNCs, respectively). 


\section{Results}

Generating and characterizing human microglia-like cells from umbilical cord blood-derived mononuclear cells (CB-iMGs)

We adapted previously reported methods ${ }^{28,29}$ for generating iMGs from adult-derived PBMCs to reprogram umbilical CB-MNCs from neonates of SARS-CoV-2 negative $(n=4)$ and SARS-CoV-2 positive $(n=2)$ mothers, delivered between 7 June 2020 and 6 July 2020 . After 2 weeks of cytokine exposure, analogous to PBMCderived iMGs, CB-iMGs displayed typical ramified microglial morphology (Fig. 1a, i) and stained positive for canonical microglial markers IBA1, CX3CR1, PU.1, and P2RY12 (Fig. 1a, ii-v), demonstrating that iMGs can be generated from umbilical cord blood-derived CB-MNCs. These cells exhibit morphologic and features and markers of cell identity comparable to those we have demonstrated for adult blood-derived PBMCs (Fig. 1b, i-v). Quantitation of immunopositive cells for microglial markers (Fig. 1c) and upregulation of canonical microglial genes (Fig. 1d) indicate comparable efficiency of iMG transdifferentiation by PBMCs and CB-MNCs.

\section{CB-iMGs demonstrate capacity to engulf isolated synaptic} material in an in vitro model of synaptic pruning

We next characterized CB-iMG function in a model of synaptic pruning using highly purified isolated nerve terminals (synaptosomes), allowing quantitation of synaptic engulfment in vitro with greater signal-to-noise than intact neural cultures ${ }^{28,29}$. Figure 2 a illustrates the real-time imaging-based phagocytosis assay workflow with CB-iMG cultures upon addition of iPSC-derived neural culture purified synaptosomes labeled with pHrodo Red SE, a pH-sensitive dye that fluoresces upon localization to lysosomes post-engulfment. Engulfment of synaptosomes can be robustly quantified in real-time live imaging (Fig. 2b, c) as well as by endpoint confocal microscopy of fixed immunostained cells ${ }^{28,29}$.

To characterize kinetics of phagocytosis over time, pHrodo-labeled synaptosomes were added to the CBiMG cultures and engulfment was visualized every $45 \mathrm{~min}$ for approximately $5 \mathrm{~h}$ using real-time live fluorescence imaging (Fig. 3a). Phagocytotic index was determined at each time point by measuring $\mathrm{pHrodo}$ area per cell (Fig. 3b) using CellProfiler as described in "Methods" and Supplementary Information. CB-iMGs demonstrate robust phagocytosis of synaptosomes, with a time course of synaptosome engulfment rising over time qualitatively similar to that observed with adult PBMCderived iMGs ${ }^{28,29}$.

\section{CB-IMGs derived from CB-MNCs exposed to maternal SARS-CoV-2 infection}

To illustrate the application of these models to study maternal exposures, we generated CB-iMGs from umbilical cord blood of neonates from mothers who tested positive for SARS-CoV-2 $(n=2)$. As observed above for CB-iMGs derived from SARS-CoV-2 unexposed pregnancies, these cells also display ramified and amoeboid morphology by phase contrast imaging (Fig. 4a), express microglial-specific markers IBA1, CX3CR1, PU.1, and P2RY12 by immunostaining (Fig. $4 \mathrm{~b}, \mathrm{i}-\mathrm{iv}$ ), and engulf
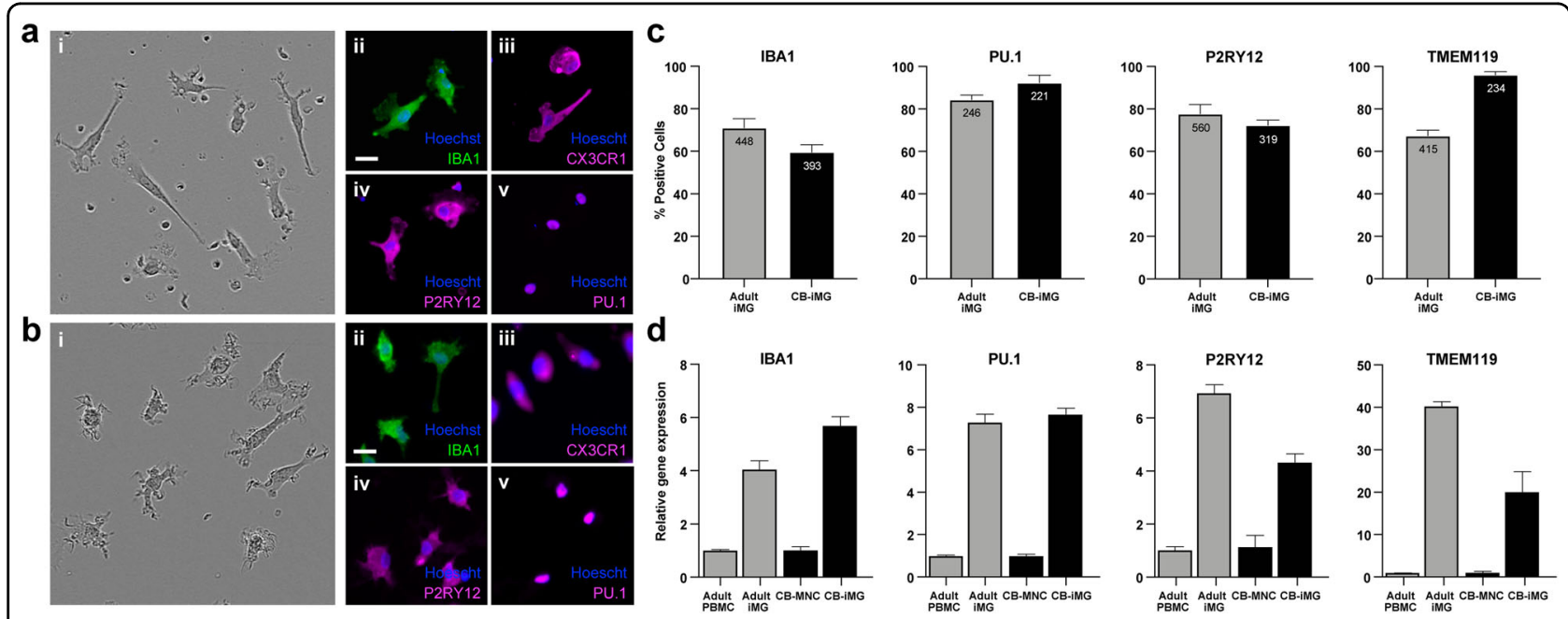

Fig. 1 Characterization of monocyte-derived induced microglia-like cells (iMGs) by direct cytokine reprogramming. a Umbilical cord monocyte-derived CB-iMGs. b Adult PBMC-derived iMGs. (i) Morphology by phase contrast; immunostained images of iMG cells stained with nuclei (Hoechst) and indicated microglial markers (ii) IBA1, (iii) CX3CR1, (iv) P2RY12, and (v) PU.1. Scale bar: $30 \mu \mathrm{m}$. c Quantitation of positively immunostained cells as a percentage of total cells (nuclei) for indicated microglial markers. Bars indicate mean of indicated no. of cells measured, error bars indicating SEM. d Quantitation of fold increase in gene expression of indicated microglial markers normalized to input (PBMCs and CB-MNCs, respectively) after iMG reprogramming, error bars indicating SEM ( $n=3$ measurements). 


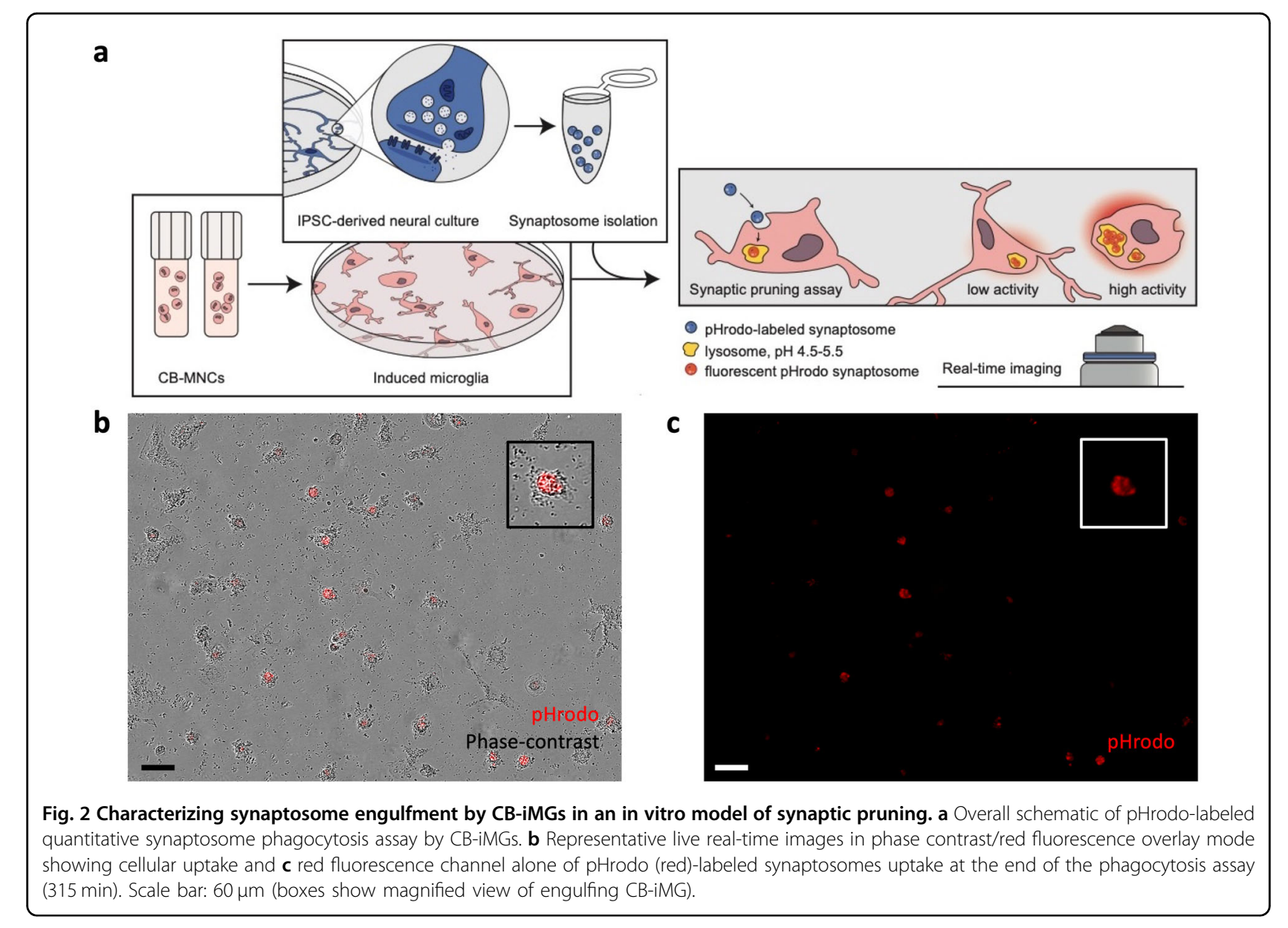

pHrodo-labeled synaptosomes in our synaptic pruning assay as visualized by real-time live imaging (Fig. $5 \mathrm{a}-\mathrm{c}$ ) and quantification (Fig. 5d). These results demonstrate the capacity to create phenotypically characteristic and functionally active CB-iMGs in a quantitative model of synaptic pruning to provide for further investigations into potential effects of maternal exposures such as SARS-CoV-2.

\section{Discussion}

Our results demonstrate the successful creation of neonatal patient-specific models of microglia-mediated synaptic pruning via cellular reprogramming of neonatal cord blood mononuclear cells. These models should facilitate novel insights into fetal brain development in the setting of maternal exposures, including but not limited to SARS-CoV-2 infection. Both SARS-CoV-2exposed and -unexposed umbilical cord blood-derived microglia-like cells express canonical microglial markers IBA1, CX3CR1, PU.1, and P2RY12, and demonstrate a range of morphologies with varying degrees of ramification, potentially reflecting a range of activation states that can be perturbed in experimental systems. Importantly, the induced microglia phagocytose synaptosomes demonstrating that they can recapitulate this key function of microglia in the developing brain. This work suggests the potential for umbilical blood mononuclear cells to serve as a non-invasive, personalized biomarker of fetal brain microglial priming. To our knowledge, microglia have not previously been modeled from umbilical cord blood or used to predict neurodevelopmental vulnerability at a time when there is a window for intervention. These models provide the potential for quantifiable endpoints that can be used to assess microglial programming in the setting of various maternal exposures, and later can be used to test the efficacy of potential therapies to ameliorate in utero priming of microglia toward a proinflammatory phenotype ${ }^{38}$.

Previous studies have demonstrated the impact of various maternal exposures, including maternal stress, metabolic disorders, air pollution, and infections, on fetal brain development ${ }^{2-4,7}$. Such exposures may lead to offspring neurodevelopmental morbidity via maternal immune activation ${ }^{39-46}$, which results in aberrant microglial programming in the developing brain ${ }^{12-15,18}$. In turn, maternal immune activation models have pointed to aberrant differentiation of fetal microglia and 
dysregulation of cytokine networks as key mechanisms underlying abnormal fetal brain development, with microglia primed toward a pro-inflammatory phenotype and altered synaptic pruning implicated in offspring morbidity ${ }^{11-15}$. Given the extent of synapse formation and

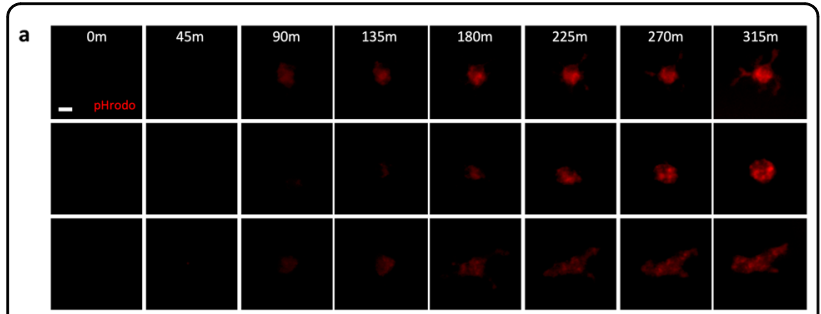

b

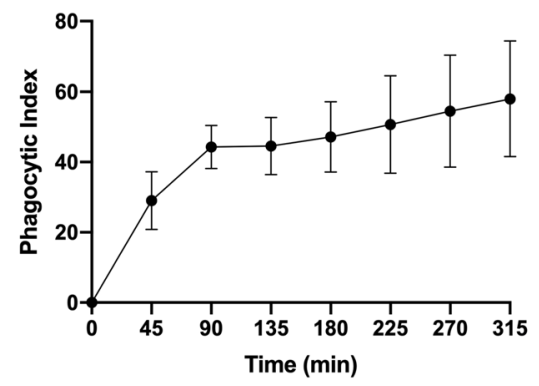

Fig. 3 Quantifying synaptosome engulfment by CB-iMGs using live real-time imaging. a Representative pHrodo (red)-labeled synaptosome engulfment in iMG cells during live real-time imaging used for quantification Scale bar: $20 \mu \mathrm{m}$. b Quantification of labeled synaptosome uptake by CB-iMGs cells during live imaging. The phagocytic index represents the mean pHrodo + area per iMG cell over $N=8$ fields per well $\times 3$ wells per line and $N=4$ separate healthy control CB-IMG line derivations. Error bars represent SEM. pruning that occurs in fetal and neonatal life, developmental microglial function represents a critical target for investigation.

We have previously demonstrated feasibility for the concept of using other monocyte populations to model microglial behavior using another monocyte type, fetal placental macrophages or Hofbauer cells, as a potential biologic surrogate for fetal microglial function in preclinical models of maternal obesity ${ }^{16}$, and have shown that maternal obesity-associated inflammation primes both fetal brain microglia and resident placental macrophages toward a highly correlated pro-inflammatory phenotype ${ }^{16}$. Personalized assays using more readily available cord blood mononuclear cells, as presented here, can be used to assess both baseline microglial function, and behavior in response to "second hit" inflammatory stimuli, which will be helpful in informing risk assessments for an individual fetus.

The COVID-19 pandemic, with its associated maternal immune activation and pro-inflammatory cytokinemediated physiology ${ }^{47,48}$, may pose risk to the developing fetal brain. While current data suggest that vertical transmission of SARS-CoV-2 is relatively rare $^{49-51}$, the profound immune activation observed in a subset of infected individuals suggests that, even if the virus itself does not cross placenta, the developing fetal brain may be impacted by maternal inflammation and altered cytokine expression during key developmental windows ${ }^{52-54}$. In this work, we suggest a model system that may be applied to investigate risk associated with the COVID-19 pandemic. Extending our work with adult peripheral blood mononuclear cells to cord blood-derived microglial a

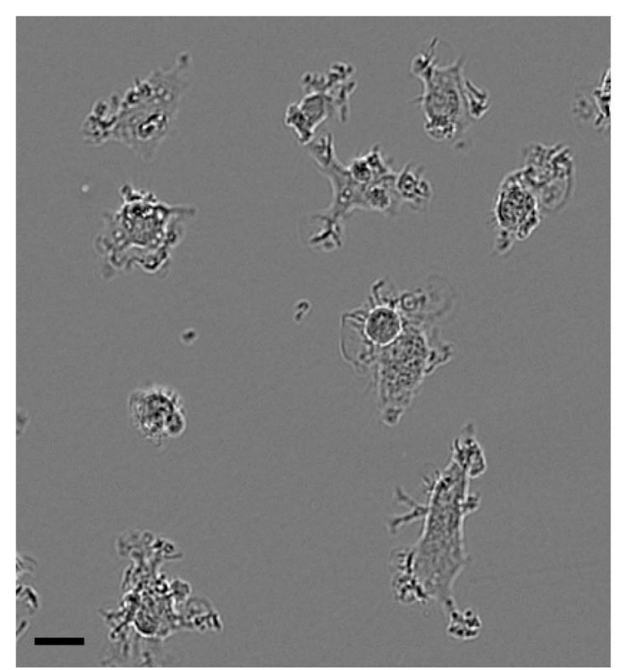

b
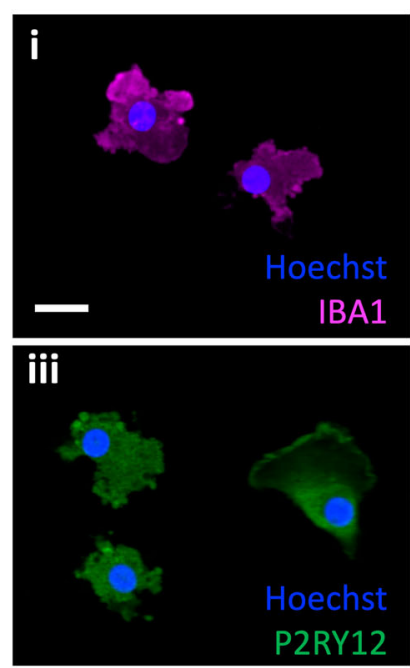

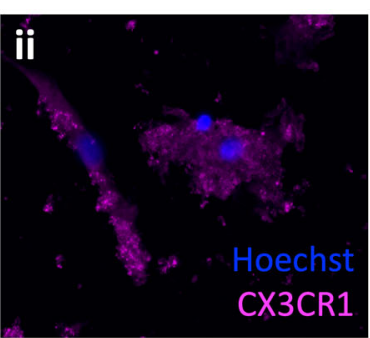

iv

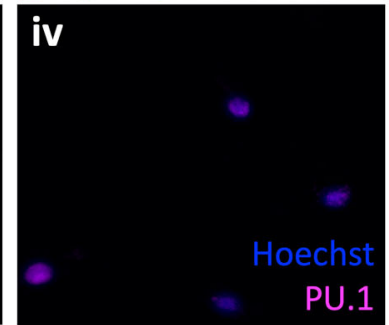

Fig. 4 Phenotypic characterization of CB-iMGs derived from CB-MNCs exposed to maternal SARS-CoV-2 infection. a Phase contrast ramified morphology of patient-derived CB-iMGs. Scale bar: $30 \mu \mathrm{m}$. b Immunostained images of exposed CB-iMG cells with nuclei (Hoechst) and indicated microglial markers (i) IBA1, (ii) CX3CR1, (iii) P2RY12, and (iv) PU.1. Scale bar: $25 \mu \mathrm{m}$. 
a

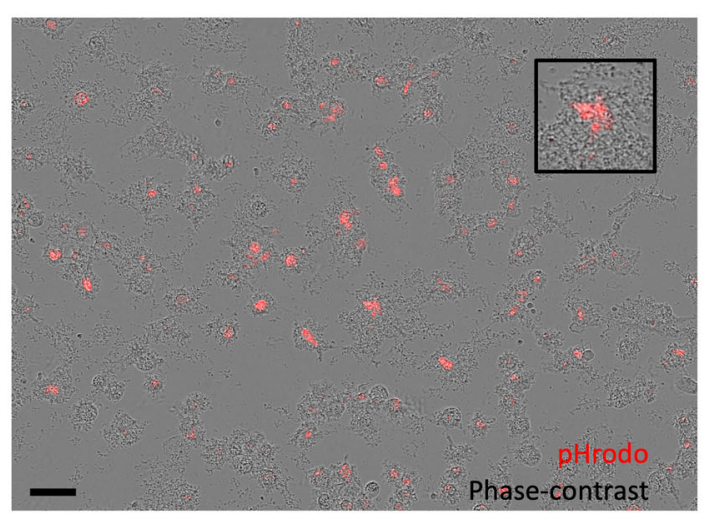

b

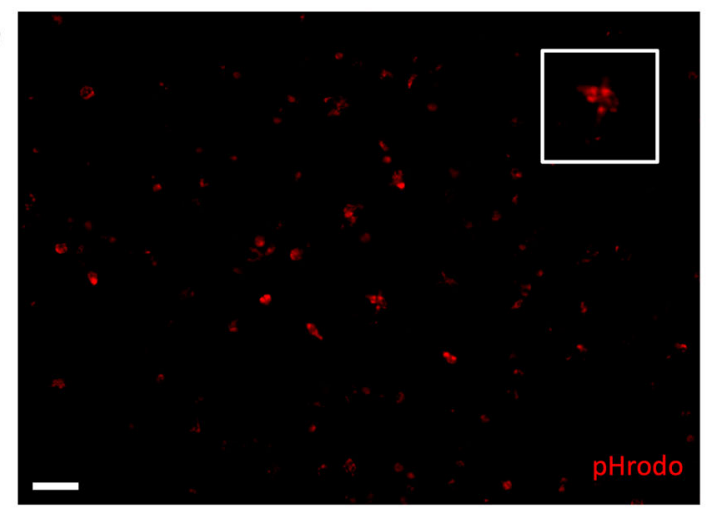

c
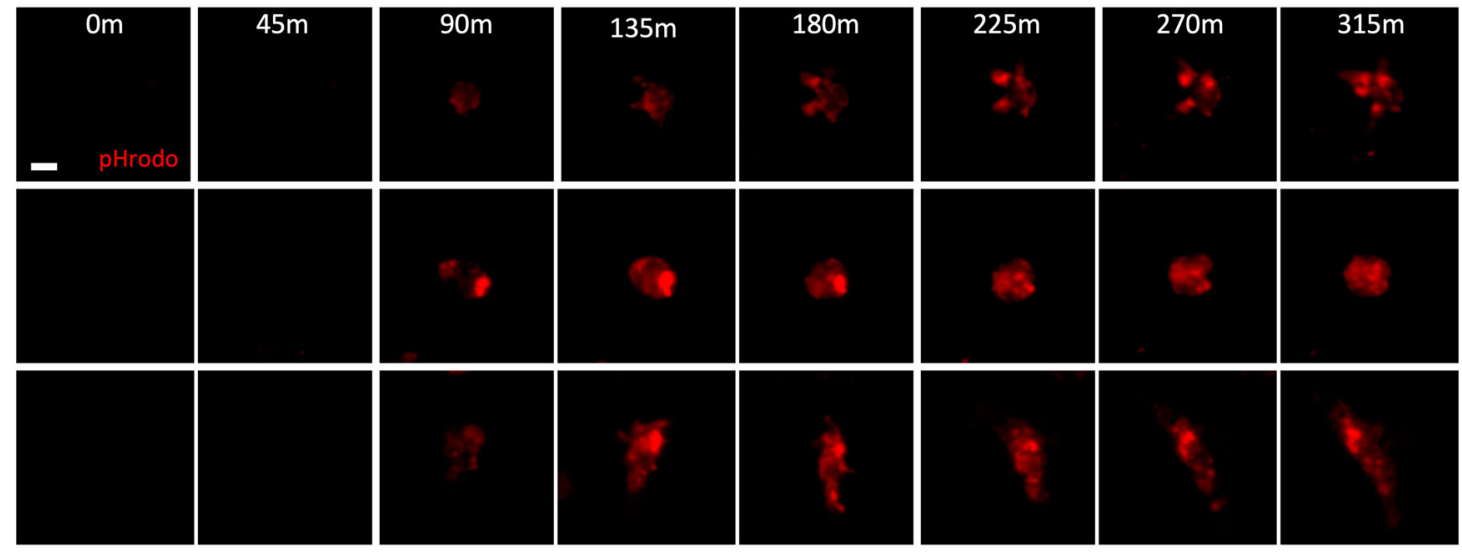

d

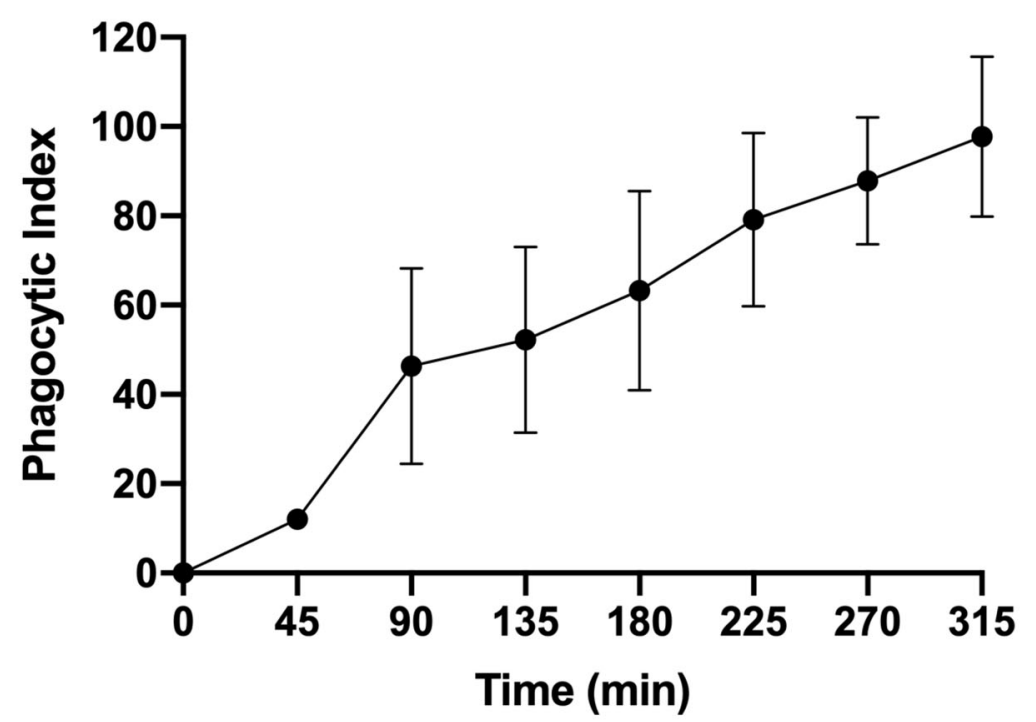

Fig. 5 Functional characterization of synaptosome engulfment by CB-iMGs derived from CB-MNCs exposed to maternal SARS-CoV-2 infection. a Representative live real-time images in phase contrast/red fluorescence overlay mode showing cellular uptake and $\mathbf{b}$ red fluorescence channel alone of pHrodo (red)-labeled synaptosomes uptake at the end of the phagocytosis assay (315 min). Scale bar: $60 \mu \mathrm{m}$ (boxes show the magnified view of engulfing (B-iMG). c Representative pHrodo (red)-labeled synaptosome engulfment in exposed CB-iMGs during live real-time imaging used for quantification. Scale bar: $20 \mu \mathrm{m}$. d Quantification of labeled synaptosome uptake by exposed CB-iMGs cells during live imaging. The phagocytic index represents the mean pHrodo+ area per iMG cell over $N=8$ fields per well $\times 3$ wells per line and $N=2$ separate $S A R S-C o V-2-$ exposed CB-IMG line derivations. Error bars represent SEM. 
models will allow for rapid, scalable models to investigate risk, yielding non-invasive, personalized assays of the impact of SARS-CoV-2 on fetal brain microglial priming and synaptic pruning function. This approach can complement more traditional approaches that will require large longitudinal cohort studies and may require years or even decades (in the case of schizophrenia, for example) to fully capture risk. The ability to detect priming of fetal brain microglia toward a pro-inflammatory phenotype extends beyond SARS-CoV-2 to include numerous other maternal infections in pregnancy, as well as the myriad maternal exposures that have been suggested to impact fetal microglial development.

In sum, we demonstrate the potential for umbilical cord blood mononuclear cells to serve as a non-invasive, personalized model of fetal brain microglial priming. These models provide the potential for quantifiable endpoints that can be used to assess microglial programming in the setting of various maternal exposures. We determined that CB-iMGs can recapitulate microglial characteristics and function in vitro, providing key insight into cells from the neonatal brain that are otherwise inaccessible at birth and throughout childhood. We illustrated their application to investigate effects of maternal infection, including SARS-CoV-2, on the developing brain $^{28}$. Beyond characterizing any consequent abnormalities, the scalability of this approach may enable investigation of targeted therapeutic strategies to rescue such dysfunction.

\section{Acknowledgements}

We are grateful to Dana Cvrk, CNM; Muriel Schwinn, NP; Lydia Shook, MD; Adeline Boatin, MD, MPH; Robin Azevedo, RN; Laurel Gardner, RN; Suzanne Stanton, RN; Natalie Croul, BA; Nicola Young, BA; and Samantha Devane, BS for their assistance with recruitment and sample collection; to all members of the MGH Obstetric-Pediatric COVID-19 Biorepository Processing Team for their assistance with sample processing and storage, to Lael Yonker, MD and Alessio Fasano, MD for their partnership on the Obstetric-Pediatric biorepository; to Jon Li, MD and $\mathrm{Xu} Y \mathrm{Yu}, \mathrm{MD}$ for critical infrastructural and regulatory support; and to Anjali Kaimal, MD,MAS, and Jeffrey Ecker, MD, for assistance with study infrastructure and departmental support. Most importantly, we thank the participants for being part of the study. This work was supported by R01MH120227 (to R.H.P.) and R01HD100022 and 3R01HD100022-02S2 (to A.G.E.).

\begin{abstract}
Author details
${ }^{1}$ Department of Psychiatry, Harvard Medical School, Boston, MA, USA. ${ }^{2}$ Center for Genomic Medicine and Department of Psychiatry, Massachusetts General Hospital, Boston, MA, USA. ${ }^{3}$ Obstetrics, Gynecology, and Reproductive Biology, Harvard Medical School, Boston, MA, USA. ${ }^{4}$ Vincent Center for Reproductive Biology, Massachusetts General Hospital, Boston, MA, USA. ${ }^{5}$ Department of Physiology and Pharmacology, Karolinska Institutet, Stockholm, Sweden
\end{abstract}

\section{Conflict of interest}

R.H.P. has received personal fees from Burrage Capital, RID Ventures, Genomind, Takeda, Psy Therapeutics, and Outermost Therapeutics, unrelated to the work described. He holds equity in Psy Therapeutics and Outermost Therapeutics. The other authors have declared no competing financial interests in relation to the work described.

\section{Publisher's note}

Springer Nature remains neutral with regard to jurisdictional claims in published maps and institutional affiliations.

Supplementary information The online version contains supplementary material available at https://doi.org/10.1038/s41398-021-01287-w.

Received: 5 January 2021 Accepted: 3 February 2021

Published online: 19 March 2021

\section{References}

1. Adams Waldorf, K. M. \& McAdams, R. M. Influence of infection during pregnancy on fetal development. Reproduction 146, R151-R162 (2013).

2. Al-Haddad, B. J. S. et al. Long-term risk of neuropsychiatric disease after exposure to infection in utero. JAMA Psychiatry 76, 594-602 (2019).

3. Al-Haddad, B. J. S. et al. The fetal origins of mental illness. Am. J. Obstet. Gynecol. 221, 549-562 (2019).

4. Bilbo, S. D., Block, C. L., Bolton, J. L., Hanamsagar, R. \& Tran, P. K. Beyond infection - maternal immune activation by environmental factors, microglial development, and relevance for autism spectrum disorders. Exp. Neurol. 299 (Pt A), 241-251 (2018).

5. Gregor, M. F. \& Hotamisligil, G. S. Inflammatory mechanisms in obesity. Annu Rev. Immunol. 29, 415-445 (2011).

6. Wilson, R. M. \& Messaoudi, I. The impact of maternal obesity during pregnancy on offspring immunity. Mol. Cell Endocrinol. 418(Pt 2), 134-142 (2015).

7. Cordeiro, C. N., Tsimis, M. \& Burd, I. Infections and brain development. Obstet. Gynecol. Surv. 70, 644-655 (2015).

8. Yockey, L. J., Lucas, C. \& Iwasaki, A. Contributions of maternal and fetal antiviral immunity in congenital disease. Science 368, 608-612 (2020).

9. Zerbo, O. et al. Maternal infection during pregnancy and autism spectrum disorders. J. Autism Dev. Disord. 45, 4015-4025 (2015).

10. Mednick, S. A. Adult schizophrenia following prenatal exposure to an influenza epidemic. Arch. Gen. Psychiatry 45, 189 (1988).

11. Nunez, J. L., Alt, J. J. \& McCarthy, M. M. A novel model for prenatal brain damage. II. Long-term deficits in hippocampal cell number and hippocampaldependent behavior following neonatal GABAA receptor activation. Exp. Neurol. 181, 270-280 (2003).

12. Nakai, Y. et al. Apoptosis and microglial activation in influenza encephalopathy. Acta Neuropathol. 105, 233-239 (2003).

13. Smolders, S., Notter, T., Smolders, S. M. T., Rigo, J. M. \& Brone, B. Controversies and prospects about microglia in maternal immune activationmodels for neurodevelopmental disorders. Brain Behav. Immun. 73, 51-65 (2018).

14. Fernandez de Cossio, L., Guzman, A., van der Veldt, S. \& Luheshi, G. N. Prenatal infection leads to ASD-like behavior and altered synaptic pruning in the mouse offspring. Brain Behav. Immun. 63, 88-98 (2017).

15. Zhao, Q. et al. Maternal immune activation-induced PPARgamma-dependent dysfunction of microglia associated with neurogenic impairment and aberrant postnatal behaviors in offspring. Neurobiol. Dis. 125, 1-13 (2019).

16. Edlow, A. G. et al. Placental macrophages, a window into fetal microglial function in maternal obesity. Int. J. Dev. Neurosci. 77, 60-68 (2019).

17. Lenz, K. M. \& McCarthy, M. M. A starring role for microglia in brain sex differences. Neuroscientist 21, 306-321 (2015).

18. Bilimoria, P. M. \& Stevens, B. Microglia function during brain development, new insights from animal models. Brain Res. 1617, 7-17 (2015).

19. Paolicelli, R. C. et al. Synaptic pruning by microglia is necessary for normal brain development. Science 333, 1456-1458 (2011).

20. Schafer, D. P. et al. Microglia sculpt postnatal neural circuits in an activity and complement-dependent manner. Neuron 74, 691-705 (2012).

21. Sierra, A. et al. Microglia shape adult hippocampal neurogenesis through apoptosis-coupled phagocytosis. Cell Stem Cell 7, 483-495 (2010).

22. Ginhoux, F. et al. Fate mapping analysis reveals that adult microglia derive from primitive macrophages. Science 330, 841-845 (2010).

23. Gomez Perdiguero, E. et al. Tissue-resident macrophages originate from yolksac-derived erythro-myeloid progenitors. Nature 518, 547-551 (2015).

24. Ginhoux, F. \& Prinz, M. Origin of microglia: current concepts and past controversies. Cold Spring Harb. Perspect. Biol. 7, a020537 (2015).

25. Gomez Perdiguero, E., Schulz, C. \& Geissmann, F. Development and homeostasis of "resident" myeloid cells: the case of the microglia. Glia $61,112-120$ (2013). 
26. Haley, M. J., Brough, D., Quintin, J. \& Allan, S. M. Microglial priming as trained immunity in the brain. Neuroscience 405, 47-54 (2019).

27. Merad, M. \& Martin, J. C. Pathological inflammation in patients with COVID-19: a key role for monocytes and macrophages. Nat. Rev. Immunol. 20, 355-362 (2020).

28. Sellgren, C. M. et al. Increased synapse elimination by microglia in schizophrenia patient-derived models of synaptic pruning. Nat. Neurosci. 22, 374-385 (2019).

29. Sellgren, C. M. et al. Patient-specific models of microglia-mediated engulfment of synapses and neural progenitors. Mol. Psychiatry 22, 170-177 (2017).

30. Baum, M. L. et al. CUB and Sushi Multiple Domains 1 (CSMD1) opposes the complement cascade in neural tissues. Preprint at bioRxiv, https:/doi.org/ 10.1101/2020.09.11.291427 (2020).

31. Lui, H. et al. Progranulin deficiency promotes circuit-specific synaptic pruning by microglia via complement activation. Cell 165, 921-935 (2016).

32. Sarn, N. et al. Cytoplasmic-predominant Pten increases microglial activation and synaptic pruning in a murine model with autism-like phenotype. Mol. Psychiatry https://doi.org/10.1038/s41380-020-0681-0 (2020).

33. Boyum, A. Isolation of lymphocytes, granulocytes and macrophages. Scand. J. Immunol. Suppl 5, 9-15 (1976)

34. Gray, E. G. \& Whittaker, V. P. The isolation of nerve endings from brain: an electron-microscopic study of cell fragments derived by homogenization and centrifugation. J. Anat. 96, 79-88 (1962).

35. Kamat, P. K., Kalani, A. \& Tyagi, N. Method and validation of synaptosomal preparation for isolation of synaptic membrane proteins from rat brain. MethodsX 1, 102-107 (2014).

36. Tenreiro, P. et al. Comparison of simple sucrose and percoll based methodologies for synaptosome enrichment. Anal. Biochem. 517, 1-8 (2017).

37. McQuin, C. et al. CellProfiler 3.0: next-generation image processing for biology. PLoS Biol. 16, e2005970 (2018).

38. Mohammadi, A., Esmaeilzadeh, E., Li, Y., Bosch, R. J. \& Li, J. Z. SARS-CoV-2 detection in different respiratory sites: a systematic review and meta-analysis. EBioMedicine 59, 102903 (2020).

39. Bergdolt, L. \& Dunaevsky, A. Brain changes in a maternal immune activation model of neurodevelopmental brain disorders. Prog. Neurobiol. 175, 1-19 (2019).

40. Careaga, M., Murai, T. \& Bauman, M. D. Maternal immune activation and autism spectrum disorder: from rodents to nonhuman and human primates. Biol. Psychiatry 81, 391-401 (2017).
41. Haddad, F. L., Patel, S. V. \& Schmid, S. Maternal immune activation by Poly I:C as a preclinical model for neurodevelopmental disorders: a focus on autism and schizophrenia. Neurosci. Biobehav. Rev. 113, 546-567 (2020).

42. Ito, H. T., Smith, S. E., Hsiao, E. \& Patterson, P. H. Maternal immune activation alters nonspatial information processing in the hippocampus of the adult offspring. Brain Behav. Immun. 24, 930-941 (2010).

43. Malkova, N. V., Yu, C. Z., Hsiao, E. Y., Moore, M. J. \& Patterson, P. H. Maternal immune activation yields offspring displaying mouse versions of the three core symptoms of autism. Brain Behav. Immun. 26, 607-616 (2012).

44. Brown, A. S. \& Meyer, U. Maternal immune activation and neuropsychiatric illness: a translational research perspective. Am. J. Psychiatry 175, 1073-1083 (2018).

45. Conway, F. \& Brown, A. S. Maternal immune activation and related factors in the risk of offspring psychiatric disorders. Front. Psychiatry 10, 430 (2019).

46. Missault, S. et al. The risk for behavioural deficits is determined by the maternal immune response to prenatal immune challenge in a neurodevelopmental model. Brain Behav. Immun. 42, 138-146 (2014).

47. Liu, J. et al. Longitudinal characteristics of lymphocyte responses and cytokine profiles in the peripheral blood of SARS-CoV-2 infected patients. EBioMedicine 55, 102763 (2020).

48. Mehta, P. et al. COVID-19: consider cytokine storm syndromes and immunosuppression. Lancet 395, 1033-1034 (2020).

49. Kotlyar, A. M. et al. Vertical transmission of coronavirus disease 2019: a systematic review and meta-analysis. Am. J. Obstet. Gynecol. 224, 35-53.e3 (2021).

50. Flaherman, V. J. et al. Infant outcomes following maternal infection with SARS CoV-2: first report from the PRIORITY Study. Clin. Infect. Dis. ciaa1411, https:// doi.org/10.1093/cid/ciaa1411 (2020).

51. Vivanti, A. J. et al. Transplacental transmission of SARS-CoV-2 infection. Nat. Commun. 11, 3572 (2020).

52. Cai, Z., Pan, Z. L., Pang, Y., Evans, O. B. \& Rhodes, P. G. Cytokine induction in fetal rat brains and brain injury in neonatal rats after maternal lipopolysaccharide administration. Pediatr. Res. 47, 64-72 (2000).

53. Stevens, B. et al. The classical complement cascade mediates CNS synapse elimination. Cell 131, 1164-1178 (2007).

54. Urakubo, A., Jarskog, L. F., Lieberman, J. A. \& Gilmore, J. H. Prenatal exposure to maternal infection alters cytokine expression in the placenta, amniotic fluid, and fetal brain. Schizophr. Res. 47, 27-36 (2001). 